\title{
A METHOD OF ASCENT FOR SOLVING BOUNDARY VALUE PROBLEMS
}

\author{
BY ROBERT P. GILBERT
}

Communicated by Wolfgang Wasow, April 16, 1969

Stefan Bergman [1] and Ilya Vekua [4] have given representation formulas for solutions of the partial differential equation (1). We obtain an improvement of their results for the case of two independent variables (namely equation (2) with $n$ set equal to 2). Furthermore, we are able to extend our result to higher dimensions (the ascent) by a remarkably simple variation of this two dimensional formula. Our representation (2) also contains Vekua's formulas [4, p. 59], for the Helmholtz equation in $n \geqq 2$ variables.

THEOREM 1. Let $B\left(r^{2}\right)$ be an entire function of $r^{2}$, and $R\left(\zeta, \zeta^{*} ; z, z^{*}\right)$ be the Riemann function ${ }^{1}$ of the elliptic partial differential equation, ${ }^{2}$

$$
\Delta_{2} u+B\left(r^{2}\right) u=0, \quad r=\|x\|, \quad \mathrm{x}=\left(x_{1}, x_{2}\right) .
$$

Then the function defined by

(2) $u(\mathrm{x})=h(\mathrm{x})+\int_{0}^{1} \sigma^{n-1} G\left(r ; 1-\sigma^{2}\right) h\left(\mathrm{x} \sigma^{2}\right) d \sigma, \quad \mathrm{x}=\left(x_{1} \cdots, x_{n}\right)$

where $h(x)$ is harmonic in a star-like region (with respect to the origin) $D$, and $G\left(r, 1-\sigma^{2}\right) \equiv-2 r R_{1}\left(r \sigma^{2}, 0 ; r, r\right)$, is a solution of

$$
\Delta_{n} u+B\left(r^{2}\right) u=0,
$$

for $x \in D$. Furthermore, each regular solution of (3) may be represented in the form (2).

Proof. Using Bergman's integral operator of the first kind [1, p. 10], which generates a complete system of solutions for equation (1), namely

$$
u(x)=2 \operatorname{Re}\left\{\int_{0}^{+1} E(r, t) f\left(z\left[1-t^{2}\right]\right) \frac{d t}{\left(1-t^{2}\right)^{1 / 2}}\right\}, \quad\|x\|=r
$$

one may obtain the alternate representation,

$$
u(\mathrm{x})=h(\mathrm{x})+\sum_{l \geq 1} 2 \frac{e_{l}\left(r^{2}\right)}{B\left(l, \frac{1}{2}\right)} \int_{0}^{1} \sigma\left(1-\sigma^{2}\right)^{l-1} h\left(\sigma^{2} \mathrm{x}\right) d \sigma,
$$

1 See [2, Chapter V], [3, Chapter III], and [4].

${ }^{2} \Delta_{n} \equiv \partial^{2} / \partial x_{1}^{2}+\partial^{2} / \partial x_{2}^{2}+\cdots+\partial^{2} / \partial x_{n}^{2}$, and $z=x_{1}+i x_{2}, \quad z^{*}=x_{1}-i x_{2}, \quad \xi=\xi+i \eta_{1}$ $\zeta^{*}=\xi-i \eta$ 
where

$$
h(x) \equiv 2 \int_{0}^{1} \operatorname{Re}\left\{f\left(z\left[1-t^{2}\right]\right)\right\} \frac{d t}{\left(1-t^{2}\right)^{1 / 2}}
$$

and

$$
e_{l}\left(r^{2}\right)=\frac{1}{(2 l) !}\left[\frac{\partial^{2 l}}{\partial t^{2 l}} E(r, t)\right]_{t=0}
$$

Since (4) may be seen to be the real part of a solution of a complex Goursat problem (see [3, Chapter III]), it may also be represented in terms of the Riemann function of the formally hyperbolic equation which arises from substituting $z=x_{1}+i x_{2}, z^{*}=x_{1}-i x_{2}$ into (1). Such a substitution is clearly valid since $B\left(z z^{*}\right)$ is holomorphic in $\mathbf{C}^{2}$. By identifying the real part of the Riemann function representation of this Goursat problem with (5) one obtains equation (2) with $n=2$. In order to establish (2) for $n>2$ one must obtain first a generalization of (4) for $n>2$. This may be done by a representation of the form

$$
u(\mathrm{x})=\int_{0}^{1} t^{n-2} E(r, t ; n) H\left(\mathrm{x}\left[1-t^{2}\right]\right) \frac{d t}{\left(1-t^{2}\right)^{1 / 2}},
$$

where $E(r, t ; n)$ is a solution of

$$
\left(1-t^{2}\right) E_{r t}+(n-3)\left(t^{-1}-t\right) E_{r}
$$

$$
+r t\left(E_{r r}+\frac{n-2}{r} E_{r}+B E\right)=0
$$

which satisfies

$$
\begin{gathered}
\lim _{t \rightarrow 0^{+}}\left(t^{n-3} E_{r}\right) r^{-1}=0, \quad \lim _{t \rightarrow 1^{-}}\left(\left(1-t^{2}\right)^{1 / 2} E_{r}\right) r^{-1}=0, \\
\lim _{r \rightarrow 0^{+}} E=0 .
\end{gathered}
$$

It may be shown using the method of majorants that such solutions exist. Representation (8) may be reformulated as

$$
u(x)=h(x)+\sum_{l \geq 1} c_{l}\left(r^{2} ; n\right) \int_{0}^{1} \sigma^{n-1}\left(1-\sigma^{2}\right)^{l-1} h\left(x \sigma^{2}\right) d \sigma
$$

with

$$
c_{l}\left(r^{2} ; n\right)=2 e_{l}\left(r^{2} ; n\right) \Gamma\left(l+\frac{n-1}{2}\right)\left\{\Gamma\left(\frac{n-1}{2}\right) \Gamma(n)\right\}^{-1}
$$


and where $e_{l}\left(r^{2} ; n\right)$ are the even Taylor coefficients of $E(r ; t ; n)$. The function $G(r, \tau)$ defined by

$$
G(r, \tau) \equiv \sum_{l \geq 1} c_{l}\left(r^{2} ; n\right) \tau^{l-1}
$$

is seen to satisfy the partial differential equation,

$$
2(1-\tau) G_{r r}-G_{r}+r\left(G_{r r}+B G\right)=0,
$$

and the data

$$
G(0, \tau)=0, \quad G(r, 0)=-\int_{0}^{r} r B\left(r^{2}\right) d r
$$

and is therefore independent of the dimension $n$. This proves the first part of our theorem. To realize that each solution may be written in this form we need only recognize that (2) may be rewritten as a Volterra equation by a simple change of variables.

ADDED IN PROOF. It also may be shown that (2) has the following inverse: $h(\mathrm{x})=u(\mathrm{x})+\int_{0}^{1} \sigma^{n-1} g\left(r, 1-\sigma^{2}\right) u\left(\mathrm{x} \sigma^{2}\right) d \sigma$, where $g\left(r ; 1-\sigma^{2}\right)$ $=-2 \hat{R}_{1}\left(r \sigma^{2}, 0 ; r, r\right)$, and $\hat{R}$ is the Riemann function of (1) with $B$ replaced by minus $B$.

REMARK. For the special case of Helmholtz's equation Vekua has already given such a method of ascent. Namely for $B\left(r^{2}\right) \equiv \lambda^{2}$ one has $[4$, p. 59]

$$
u(\mathrm{x})=h(\mathrm{x})-x r \int_{0}^{1} \sigma^{n-1} J_{1}\left(\lambda r\left(1-\sigma^{2}\right)^{1 / 2}\right) h\left(\mathrm{x} \sigma^{2}\right) \frac{d \sigma}{\left(1-\sigma^{2}\right)^{1 / 2}},
$$

holding for integer $n \geqq 2$. Our representation (2) contains this example as a special case, which may be seen by a simple computation.

THEOREM 2. Let $D$ be star-like with respect to the origin and $B\left(r^{2}\right)$ an entire function, such that $B\left(r^{2}\right)<0$ in $D$. Furthermore, let $\partial D$ be $a$ Lyapunov boundary and $f(x)$ a continuous function on $\partial D$. Then there exists a unique solution of (3) which may be represented as in equation (2), where $h(x)$ is given as a double layer potential

$$
h(x)=\frac{\Gamma(n / 2)}{\pi^{n / 2}} \int_{\partial D} \mu(y) \frac{\partial}{\partial \nu_{y}}\left(\frac{1}{\|x-y\|^{n-2}}\right) d \omega_{y},
$$

and $\mu(y)$ is a solution of the Fredholm integral equation, 


$$
\begin{aligned}
& f(\mathrm{x})=\mu(\mathrm{x})+\frac{\Gamma(n / 2)}{\pi^{n / 2}} \int_{\partial D} \mu(y)\left\{\frac { \partial } { \partial \nu _ { y } } \left(\frac{1}{\|\mathrm{x}-y\|^{n-2}}\right.\right. \\
&\left.-2 r \int_{0}^{1} \sigma^{n-1} R_{1}\left(r \sigma^{2}, 0 ; r, r\right) \frac{\partial}{\partial \nu_{y}}\left(\frac{1}{\left\|x \sigma^{2}-y\right\|^{n-2}}\right)\right\} d \omega_{y}, \\
& x \in \partial D .
\end{aligned}
$$

Proof. This follows by substituting the double layer potential into the representation (11), using the Fubini theorem to change orders of integration, and computing the residue as $x \rightarrow \partial D$ from the inside.

\section{REFERENCES}

1. Stefan Bergman, Integral operators in the theory of linear partial differential equations, Springer-Verlag, Berlin, 1961.

2. Paul R. Garabedian, Partial differential equations, Wiley, New York, 1964.

3. Robert P. Gilbert, Function theoretic methods in partial differential equations, Academic Press, New York, 1969. 1967.

4. Ilya N. Vekua, New methods for solving elliptic equations, Wiley, New York,

INDIANA UNIVERSITY, BLOOMINGTON, INDIANA 47401 